\title{
REPEATED TENSILE BEHAVIOUR AND DAMAGE EVOLUTION OF A 2.5D C/SiC COMPOSITE CHARACTERISED BY GREY VERHULST MODELS
}

\author{
"YIQIANG WANG*, LITONG ZHANG** \\ *College of Aeronautical Engineering, Civil Aviation University of China, \\ Tianjin 300300, People's Republic of China \\ **National Key Laboratory of Thermostructure Composite Materials, Northwestern Polytechnical University, \\ Xi'an Shaanxi, People's Republic of China \\ "E-mail: wyq80@126.com
}

Submitted June 16, 2018; accepted August 13, 2018

\begin{abstract}
Keywords: $\mathrm{C} / \mathrm{SiC}$, Tensile behaviour, Damage characteristics, Grey systems
The microstructure and repeated tensile behaviour of a $2.5 \mathrm{D} \mathrm{C/SiC} \mathrm{composite} \mathrm{fabricated} \mathrm{by} \mathrm{chemical} \mathrm{vapour} \mathrm{infiltration}$ were examined and the associated damage evolution was characterised by the residual strain and reloading modulus. Both the envelope stress-strain behaviour and the associated damage evolution of the composite can be well characterised by three regimes including the matrix cracking emergence, a multiplication followed by the matrix cracking saturation. The grey Verhulst model and the inverse grey Verhulst model were established for the evolution of the damage characteristics and the constitutive behaviour, respectively. Excellent agreement between the predictions and experimental results was obtained for both the grey models, which indicates the nature of intra-specific competition for these matrix cracks in the composite studied. Such robust relationships can be further applied to facilitate the modelling of the constitutive behaviour of other ceramic composite systems.
\end{abstract}

\section{INTRODUCTION}

Continuous carbon fibre reinforced silicon carbide ceramic matrix composites $(\mathrm{C} / \mathrm{SiC} \mathrm{CMCs})$ are one of the most promising candidates for many high temperature applications such as aerospace and aircraft thermostructural components because of their higher strength and enhanced fracture toughness $[1,2]$. Traditional 2D laminated composites have been investigated extensively [3-6]. However, their wide-spread applications in many structural components have been limited by fabricating problems and poor delamination resistance. Although 3D braided composites have improved the delamination resistance and can be used to fabricate a complex net or near-net shaped components [7], they are still expensive and not applicable for manufacturing the components with one closed end such as nose caps. To overcome these difficulties, the authors have recently developed a unique kind of multilayer $\mathrm{C} / \mathrm{SiC}$ composite, namely $2.5 \mathrm{D}$ angle-interlock woven composites. As a new class of textile composites, they have a great deal of advantages over the conventional laminated composites, including near net-shape, better out-of-plane stiffness and strength, etc. The characteristics of the weave technique make the fabric preform particularly suitable for conforming to the mould surface of the dome-shaped components.
Up to now, although $2.5 \mathrm{D}$ woven composites have been successfully applied in the structure of aero-engine fan blades [8], the studies on the mechanical behaviour of material are focused on the elastic and fatigue properties [9-12], but the reports about damage behaviour and its prediction are still limited. It is difficult to fully predict their mechanical characteristics and damage mechanism owing to their high anisotropic nature and relatively complex fibre architectures in comparison with the laminate composites. Although some research has been undertaken to investigate the mechanical behaviour of 2.5D composites based on the finite element (FE) method, the majority of the FE models have not taken the influence of the outmost layer structure into consideration $[13,14]$. Furthermore, these relatively cumbersome methods have the disadvantages of being time consuming with regards to the tasks of defining the geometry of the composite and the Representative Elementary Volume (REV), the meshing and the calculation procedures, besides the need of super computers to make the computation. However, significant amount of the researchers' attention has been attracted by the recently developed grey system theory in advanced engineering, which is more suitable for studying uncertain problems with less data and poor information. The application scope of grey system theory has successfully been extended to industry, social affairs, economy, energy, financial and other fields [15-17]. 
In order to utilise the novel composites most efficiently, a thorough understanding and the modelling of their mechanical properties is essential. Therefore, the composite microstructures were firstly characterised by the fibre's architecture. Then the uniaxial tensile behaviour of the composite was investigated under repeated loading/unloading tests and the damage was characterised by evolutions of the damage characteristics, such as the residual strain and modulus. Both the tensile behaviour and damage evolution were predicted based on the grey Verhulst models.

\section{EXPERIMENTAL}

\section{Composite fabrication}

The carbon fibre utilised was T300 (Nippon Toray corporation), and each yarn contained 6000 fibres. The preformed fibres fabricated by a proprietary 2.5-dimensional weave technique were supplied by the Nanjing Institute of Glass Fiber, People's Republic of China. The fibre volume fraction was about $40 \%$. The composite panels were processed by chemical vapour infiltration (CVI) to deposit a pyrocarbon interphase (thickness about $0.2 \mu \mathrm{m}$ ) and a $\mathrm{SiC}$ matrix. These processing steps resulted in a material having a bulk density about $2.1 \mathrm{~g} \cdot \mathrm{cm}^{-3}$ and an open porosity inherent to the CVI process in the range of 10-15 vol. \% determined by Archimedes' method.

Tension tests and microstructural observation

The dog bone-shaped tension specimens were cut from an as-processed composite panel along the warp directions, and then the specimens were sealed with a CVD SiC coating (a thickness of about $50 \mu \mathrm{m}$ ). The overall dimensions of the tension specimens are $120 \times 12 \times 3.5 \mathrm{~mm}$ with a central reduced gauge section of $30 \times 10 \times 3.5 \mathrm{~mm}$. Using an Instron 1196 test machine at a crosshead speed of $0.05 \mathrm{~mm} \cdot \mathrm{min}^{-1}$, a series of incremental loading/unloading/reloading cycles were conducted to investigate the evolution of the damage and the associated changes such as the reloading moduli and the irreversible strains of the specimens, with the loading direction parallel to the warp yarns. The stress intervals were set to about $40 \mathrm{MPa}$, i.e., the peak stress value in a loading/unloading cycle is about $40 \mathrm{MPa}$ higher than that in the preceding loading/unloading cycle. Strains were recorded with an extensometer with a gauge length of $25 \mathrm{~mm}$. Five specimens were tested to ensure the consistency of the testing results. The polished surfaces of the tested specimens were examined by optical microscopy (OM, OLYMPUS PM-T3) to determine the ultimate damages.

\section{RESULTS AND DISCUSSION}

\section{Microstructure characterisation}

The macro-mechanical performance of the composites is primarily dependent on their final weave architecture, so adequate control over their final weave architecture is of prime importance. The present $2.5 \mathrm{D}$ weave architecture is a so-called angle interlock fabric with shallow straight-joints, and the schematic of the preformed fibre architecture is shown in Figure 1. The first set of yarns that ran in the weaving direction is called the warp yarns, while the second set of yarns that ran transverse to the weaving direction is called the weft yarns. This unique kind of multilayer fabric is composed of layers of approximately straight weft yarns and a set of approximately sinusoidal warp yarns, with the adjacent layers of the weft yarns interlocked together by the warp

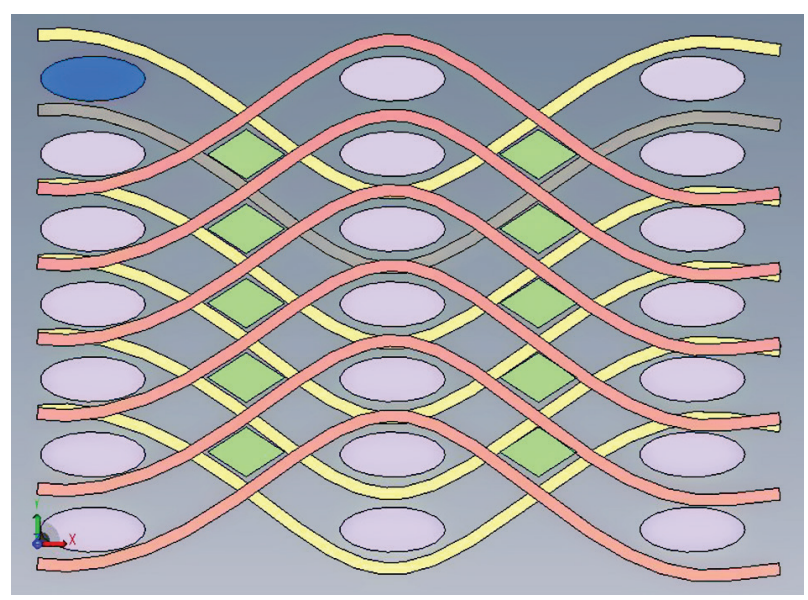

a)

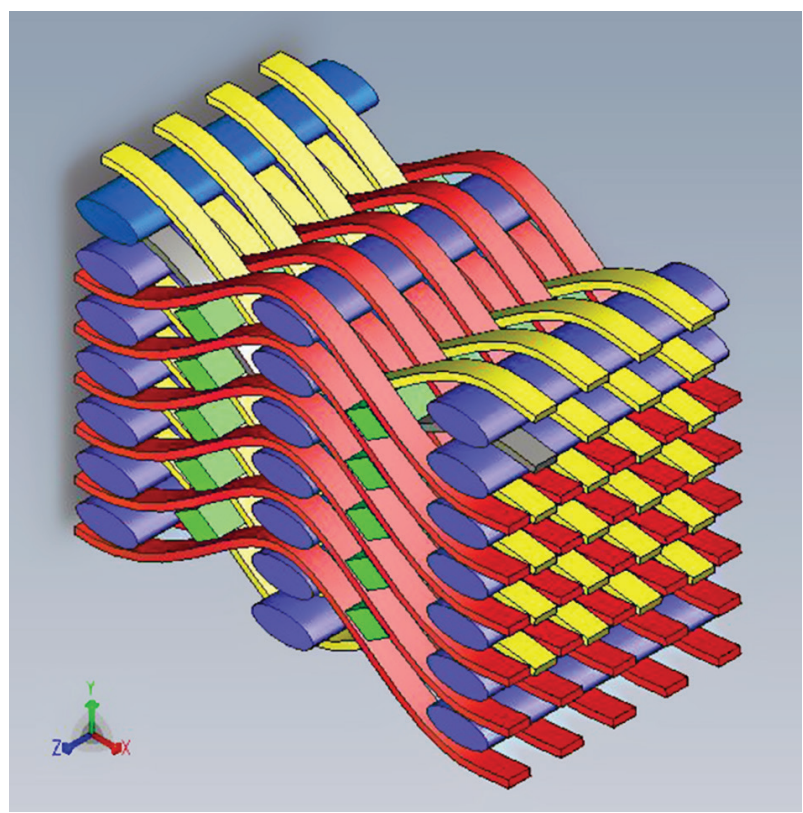

b)

Figure 1. Schematic of the 2.5D composite architecture: a) front view, b) isometric view. 
yarns. Consequently, this layer-to-layer fabric is a multilayered fabric in which the warp weavers travel from one layer to the adjacent layer, and back. The set of warp yarns woven together hold all the layers of the fabric.

The microstructure of the longitudinal section of the specimens after tensile tests is shown in Figure 2. It can be clearly seen that the weft yarns present shapes of lentils and diamonds alternately. Obviously, the warp yarns undertake dual roles. Firstly, they create the longitudinal portion which contributes mainly to the in-plane strength, and secondly, they form through-thethickness or $\mathrm{Z}$ element which contributes particularly to improved delamination resistance. The different crosssectional shapes of the weft yarns are attributed to the combination of the compaction in the graphite fixture and the symmetrical characteristics of the preformed fibre. Substantial cracks in the composites are also shown in Figure 2: transverse cracks in the weft and warp yarns, and densely spaced matrix cracks triggered by the large inter-yarn pores. These damages correspond well with the significant nonlinearity of the mechanical behaviour of the composite studied. In addition of the complex geometry, the volume fraction of the fibres and the inclination angle of the undulated warp yarns allow the tailoring of properties for specific applications. In other words, designers could produce the optimal performance of the composites for the required mechanical properties.

\section{Loading/unloading tension test}

For all the specimens from the same panel of material, the stress-strain properties show small scatters and the typical curve is shown in Figure 3. It is evident that the stress-strain loops (hysteresis behaviour) can be well defined, although the loops are relatively narrow. On reloading the specimens, the stress-strain curve does

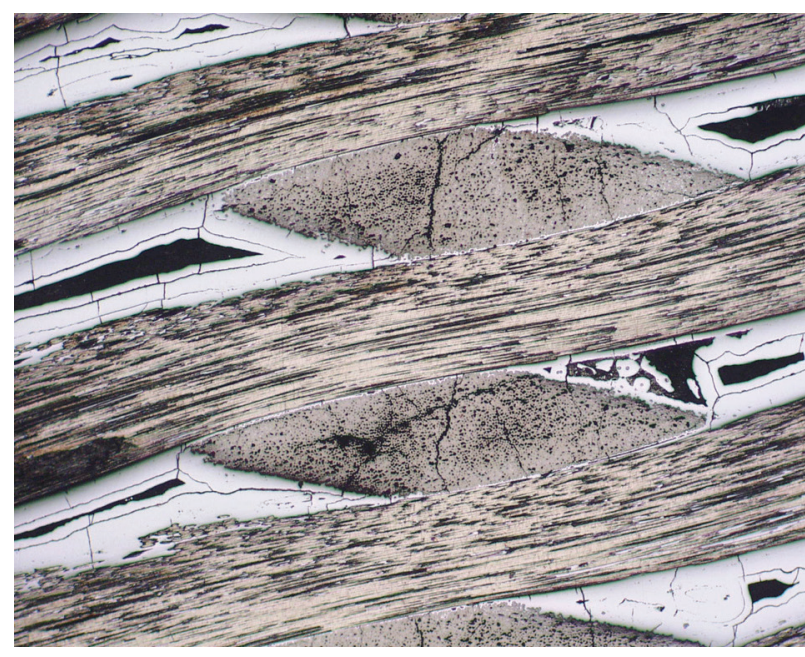

a) not show great inflection until the maximum history load of the cyclic loading test was exceeded. This implies that there are no or few new damages occurred during unloading the specimens. Consequently, the monotonic tensile behaviour can be obtained by the overall envelope of the repeated tensile stress-strain behaviour. It can be seen from the envelope that the composite behaves as a typical damageable material, exhibiting an extended non-linear stress-strain domain up to rupture.

The evolution of the damage can be characterised by the reloading moduli and residual strains. The reloading moduli were directly measured by linearly fitting the upper and lower points of the hysteresis loops due to the narrow loop width, and then they were normalised by the damage factor $D$ as follows

$$
D=\frac{E_{0}-E_{\mathrm{k}}}{E_{0}}
$$

where $E_{0}$ denotes the initial modulus, $E_{k}$ denotes the $k$-th reloading modulus.

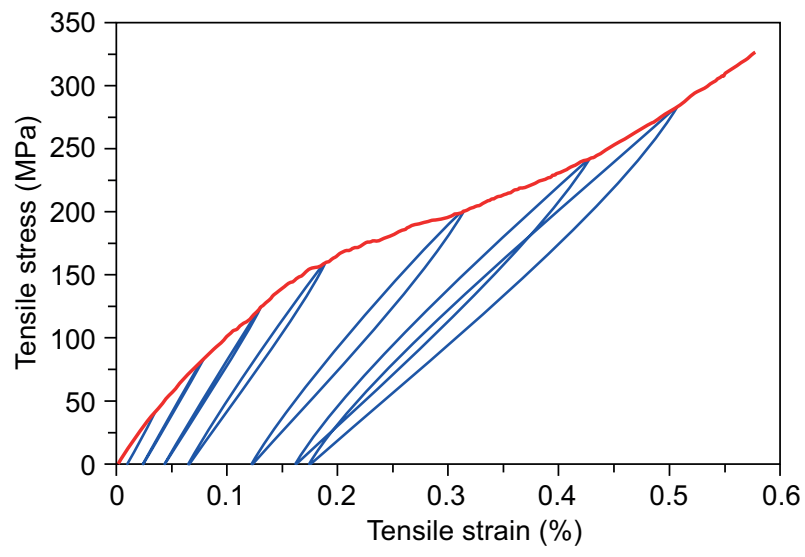

Figure 3. The representative loading/unloading tensile curve.

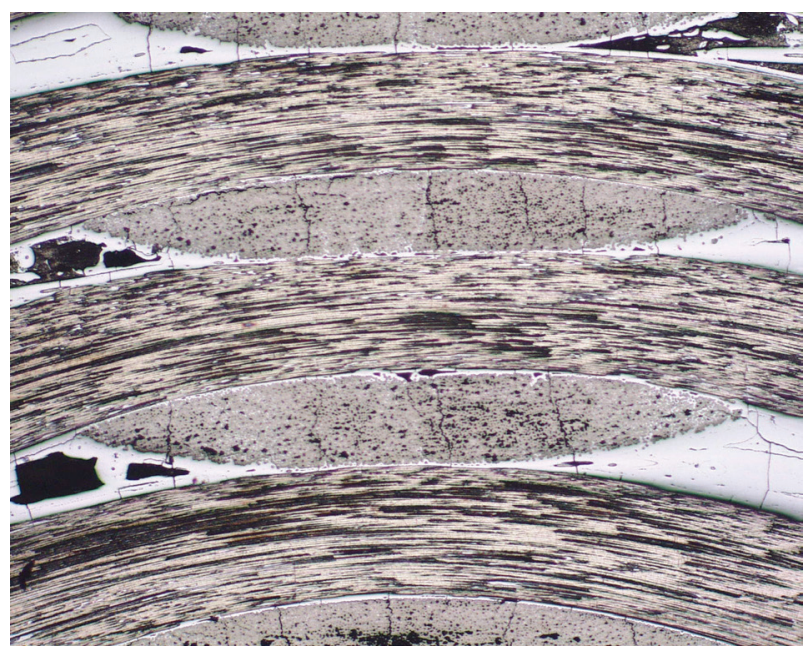

b)

Figure 2. Microstructure of the polished longitudinal section of the specimens after the tensile tests: a) transverse cracks in the diamond-shaped weft yarns, b) the lentil-shaped weft yarns. 
Both the evolutions of the residual strain and the damage factor with the applied stress are shown in Figure 4 and Figure 5, respectively. It can be seen that the evolution of the residual strain initiates almost at the onset of loading, and then increases steadily until about $100 \mathrm{MPa}$ with a subsequent dramatically increasing regime, and finally tends to saturate up to failure. The damage factor has a similar evolution, corresponding with the fact that reloading modulus decreases from the initial $160 \mathrm{GPa}$ to the final value of $85 \mathrm{GPa}$.

It is well known that the evolutions of the reloading modulus and residual strain are the direct reflection of the number of matrix cracks occurred during the loading of the CMCs. Consequently, the above-mentioned characteristics exhibited in both the evolutions of the damage factor and the residual strain are very similar

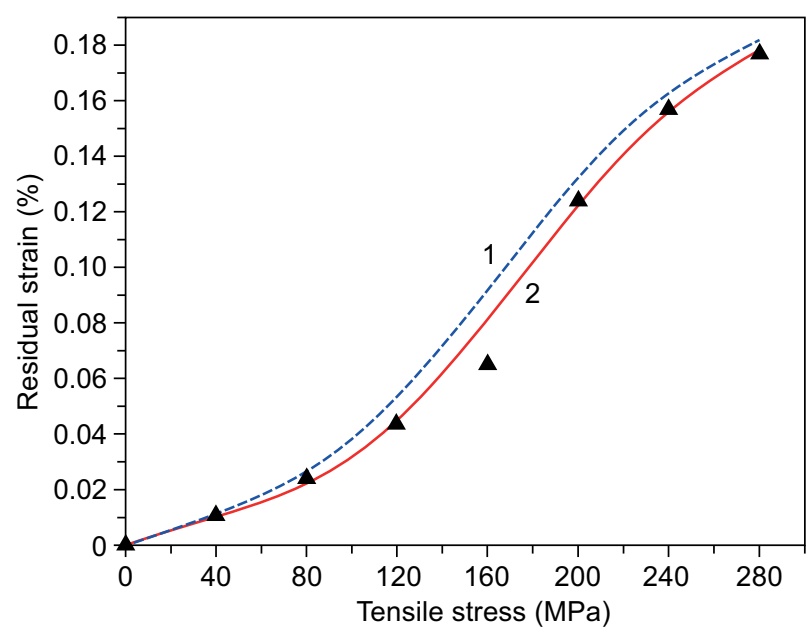

Figure 4. The evolution of the residual strain with the applied stress. The experimental data is represented by the solid triangles, while curve 1 denotes the prediction by the grey Verhulst model and curve 2 denotes the prediction with the optimised initial condition.

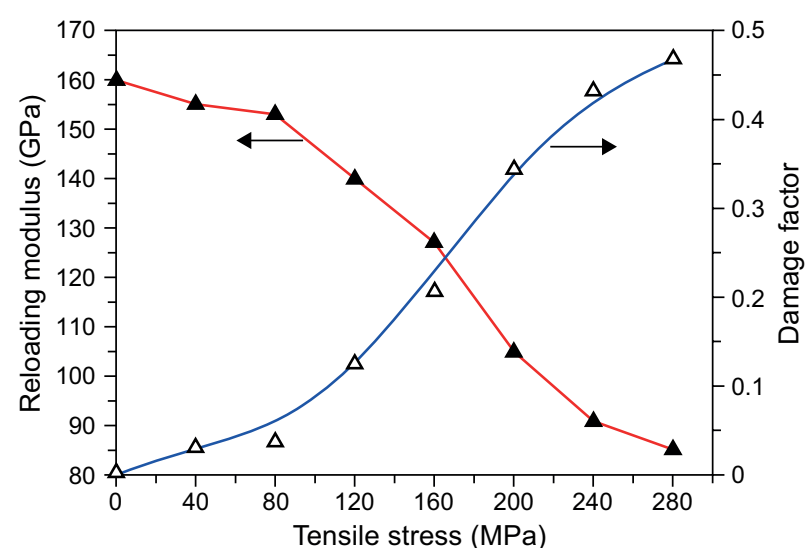

Figure 5. The degradation of the reloading modulus and corresponding damage factors. The experimental data is represented by the solid and hollow triangles. The prediction of the evolution of the damage factor by the grey Verhulst model is also shown. to the development of the self-limiting growth of a biological population, which is often described by Verhulst's equation, namely the law of population growth as follows

$$
\frac{\mathrm{d} N}{d t}=r N-\alpha N^{2}
$$

where $N$ represents the number of individuals (cracks herein) at time $t, r$ is the intrinsic growth rate, and $\alpha$ is the density-dependent crowding effect (also known as the intra-specific competition). In this equation, the population equilibrium (the so-called carrying capacity) $K$ can be derived as

$$
K=\frac{r}{\alpha}
$$

Since the residual strain is directly related to the number of matrix cracks formed, it can also be described by Verhulst's equation as follows

$$
\frac{\mathrm{d} \varepsilon^{(1)}}{d \sigma}=a \varepsilon^{(1)}-b \varepsilon^{(1)^{2}}
$$

where $\varepsilon^{(1)}$ is the residual strain, $\sigma$ is the applied stress. Thus, its grey difference equation in the discrete form can be described as

$$
\varepsilon_{(k)}^{(0)}=a z_{(k)}^{(1)}-b z_{(k)}^{(1)}{ }^{2}
$$

where $\varepsilon^{(0)}{ }_{(k)}=\varepsilon^{(1)}{ }_{(k)}-\varepsilon^{(1)}{ }_{(k-1)}, k$ is the normalised applied stress by the interval of $40 \mathrm{MPa}$, namely the sequential number of the reloading cycles. And the sequence $Z(k)$ can be obtained by using the mean consecutive neighbours operator for $\varepsilon^{(1)}$ as follows

$$
z(k)=\frac{1}{2}\left[\varepsilon_{(k)}^{(1)}+\varepsilon_{(k-1)}^{(1)}\right], \mathrm{k}=2,3, \mathrm{n} .
$$

The parameters of $a$ and $b$ can be estimated as follows by minimising the squared-errors, i.e., the least squares method:

$$
\left[\begin{array}{l}
\bar{a} \\
\widehat{b}
\end{array}\right]=\left(B^{T} B\right)^{-1} B^{T} Y
$$

where

$$
\begin{gathered}
B=\left[\begin{array}{cc}
z_{(2)}-z^{2}{ }_{(2)} \\
\ldots & \ldots \\
z_{(n)}-z^{2}{ }_{(n)}
\end{array}\right] \\
Y=\left[\begin{array}{c}
\varepsilon^{(0)}{ }_{(2)} \\
\ldots \\
\varepsilon^{(0)}{ }_{(n)}
\end{array}\right]
\end{gathered}
$$

Based on the estimated parameters, the evolution of the residual strain can be predicted by solving Equation 4, as shown below:

$$
\varepsilon^{(1)}{ }_{(k)}=\frac{a / b}{1+\left(\frac{a}{b} \cdot \frac{1}{\varepsilon^{(1)}(1)}-1\right) \mathrm{e}^{-a(k-1)}}
$$

where the selection of the initial condition of the model is arbitrary. However, in most cases, it can be simply set as $\varepsilon=\varepsilon_{(1)}^{(1)}$, namely using the first measured data point. 
Based on the measured residual strain data, the estimated parameters were calculated as $a=0.92, b=4.7$. The predicted evolution of the residual strain is shown in Figure 4 along with the experimental data. It can be seen that a slight deviation exists between the prediction and the experimental data. This is due to the improper selection of the initial condition to Equation 4, since there is no sufficient evidence that using the first set of raw data would lead to higher prediction accuracy. Therefore, the initial condition should be optimised to achieve a better agreement. The initial condition can be set as each measured data point, i.e., not only the first measured data point. Thus, the initial condition can be finally determined after comparison of the fitting degrees. As shown in Figure 4, an excellent agreement was also achieved by the predicted curve with the initial condition set as $\varepsilon=\varepsilon_{(5)}$.

The degradation of the reloading modulus and corresponding damage factors is shown in Figure 5. The prediction of the evolution of the damage factor with the parameters of $a=0.97, b=1.96$ is also shown. It can be seen that the predicted evolution exhibits excellent agreement with the experimental data. The carrying capacity (saturation value) for the residual strain and damage factor can be directly derived by the abovementioned parameters as 0.19 and 0.49 , respectively. This is in accordance with the slope recovery displayed in the envelope stress-strain curve where the remaining intact carbon fibres carry the entire load at the final regime of the matrix cracking saturation just before composite failure. The reloading modulus finally coincides with the tangent modulus, i.e., the curve slope, and achieved its saturation value according to the rule of mixture $E_{s}=E_{f} V_{f}$, where both the modulus of carbon fibres of $230 \mathrm{GPa}$ and the volume fraction were provided by the vendors.

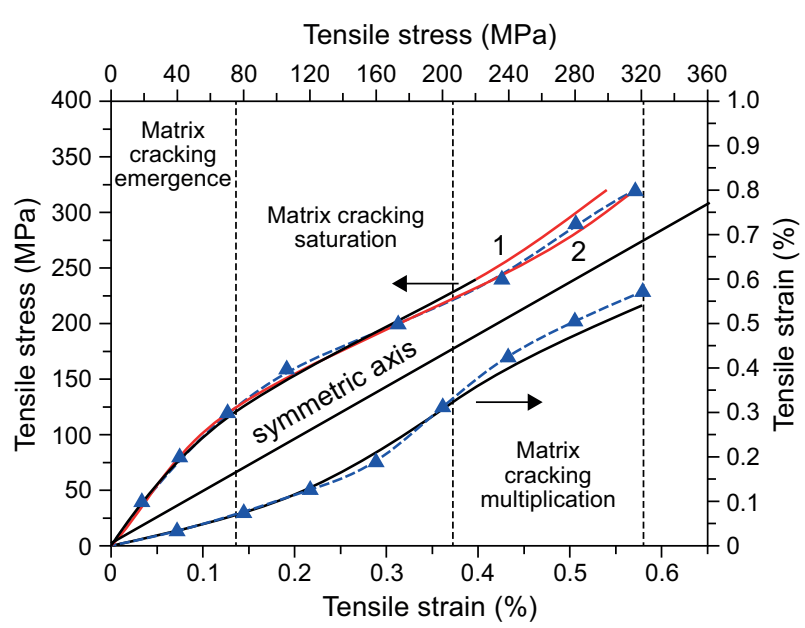

Figure 6. The prediction of the envelope strain-stress curve with the experimental data represented by the dashed line and asterisk marks. The curves of 1 and 2 stand for the predictions of the envelope stress-strain behaviour by the grey inverse Verhulst model with the optimised coefficients and the initial condition, respectively.
It is well known that the nonlinear stress-strain constitutive behaviour of the CMCs is largely due to the progressive matrix cracking. Consequently, the constitutive law of the $2.5 \mathrm{D}$ composite in this paper is also subject to Verhulst's equation, which is confirmed by the envelope strains plotted as a function of the tensile stress as shown in Figure 6. It is evident that the envelope strain-stress curve exhibits a very similar evolution to that of the damage factor or the residual strain. Therefore, the grey Verhulst method can be also used to model the constitutive law as follows

$$
\varepsilon=\frac{a / b}{1+\left(\frac{a}{b} \cdot \frac{1}{\varepsilon_{(1)}}-1\right) e^{-a\left(\sigma-\sigma_{(1)}\right)}}
$$

where the initial condition is simply set as $\varepsilon=\varepsilon_{(1)}, \sigma=\sigma_{(1)}$. The applied stress is also normalised by the equidistant loading/unloading interval of $40 \mathrm{MPa}$ to ensure the consistency.

However, the prediction accuracy cannot be guaranteed still since the selection of the initial condition is arbitrary. Therefore, Equation 11 can be rewritten as

$$
\varepsilon=\frac{1}{\beta+\alpha e^{-a\left(\sigma-\sigma_{(1)}\right)}}
$$

where the coefficients of $\alpha$ and $\beta$ reflect the selection of the initial condition which can be optimised by the leastsquared method as

$$
\min Q=\sum_{i=2}^{n}\left(\beta+\alpha e^{-a\left(\sigma_{(i)}-\sigma_{(1)}\right)}-\frac{1}{\varepsilon_{(i)}}\right)
$$

Accordingly, the coefficients of $\alpha$ and $\beta$ can be estimated as follows

$$
\left[\begin{array}{l}
\bar{a} \\
\bar{b}
\end{array}\right]=\left(B^{T} B\right)^{-1} B^{T} Y
$$

where

$$
\begin{aligned}
& B=\left[\begin{array}{cc}
e^{-a\left(\sigma_{(2)}-\sigma_{(1)}\right)} & 1 \\
\ldots & 1 \\
e^{-a\left(\sigma_{(\mathrm{n})}-\sigma_{(1)}\right)} & 1
\end{array}\right] \\
& Y=\left[\begin{array}{c}
\frac{1}{\varepsilon_{(2)}} \\
\ldots \\
\frac{1}{\varepsilon_{(\mathrm{n})}}
\end{array}\right]
\end{aligned}
$$

The predicted strain-stress curve based on the estimated coefficients of $\alpha=23.39, \beta=1.72$ and the growth rate $a=0.68$ is shown in Figure 6 , together with the experimental data. It can be seen that the prediction corresponds well with the experimental data. Although the tensile behaviour of the composite is largely nonlinear from the onset of loading, three different regimes corresponding to the evolution of the matrix cracking may be seen from the predicted strainstress curve. The first regime of the predicted curve with a slightly nonlinear behaviour corresponds to the 
matrix cracking emergence due to the presence of the processing-induced initial damages such as thermal microcracks. The middle regime of the predicted curve with a largely nonlinear behaviour corresponds to the matrix cracking multiplication due to the enormous newly formed matrix cracks and/or propagating the thickness of the $2.5 \mathrm{D}$ composites. The last regime of the predicted curve with a near plateau corresponds to the matrix cracking saturation and elongation of the remaining intact fibres, which carries the entire load. All three regimes exhibited in the predicted curve are very consistent with the evolutions of the residual strain and damage factor.

Finally, the envelope stress-strain curve can be obtained by using the inverse operation for the abovepredicted curve and is shown in Figure 6. The prediction of the final constitutive law by the inverse Verhulst Equation 16 is also shown and described as

$$
\sigma=40\left[\frac{1}{\mathrm{a}} \ln \frac{\left(a-b \varepsilon_{(5)}\right) \varepsilon}{(a-b \varepsilon)_{(5)}}+\sigma_{(5)}\right]
$$

where the initial condition was optimised and set as the fifth measured data point.

It is clear that excellent agreements were achieved by both the predicted curves, thus, validating the proposed constitutive law based on the grey Verhulst model. Moreover, the carrying capacity of the strain derived from Equation (11) is estimated as $0.65 \%$, corresponding to the infinite stress in Equation 17. This is also consistent with the observed largest ultimate tensile strain of the composite at the final failure. The parameter of growth rate $a$ of the damage factor or the residual strain is relatively larger than that of the constitutive law, indicating that the evolutions of the reloading modulus and the residual strain of the $2.5 \mathrm{D}$ composite are more susceptible to matrix cracks occurring.

\section{CONCLUSIONS}

A $2.5 \mathrm{D} \mathrm{C} / \mathrm{SiC}$ composite was fabricated by chemical vapour infiltration and its microstructure and repeated tensile behaviour were investigated, as well as the associated damage evolution. The present multilayer composite can be characterised by the angle interlock fabric with shallow straight-joints, which consists of approximately straight weft yarns with lentil and diamond shapes alternately, and approximately sinusoidal warp yarns. The largely nonlinear tensile envelope behaviour and the associated evolution of the residual strain and the reloading modulus characterised by the damage factor can be generally divided into three regimes including matrix cracking emergence, multiplication, followed by matrix cracking saturation. Both the evolution of the residual strain and the damage factor can be well predicted by the grey Verhulst models, indicating the nature of the intra-specific competition for these matrix cracks in the studied composite. The constitutive stressstrain behaviour can also be successfully predicted by the inverse grey Verhulst model. Such robust relationships can be further applied to other ceramic composite systems to establish the constitutive behaviour and optimise material parameters by the corresponding damage evolutions.

\section{Acknowledgements}

The authors would like to acknowledge the financial support of the Scientific Research Foundation of the Civil Aviation University of China under Grant No. 3122016B003, and the Civil Aviation Administration of China under Grant No. MHRD20160105.

\section{REFERENCES}

1. Naslain R. (2004): Design, preparation and properties of non-oxide $\mathrm{CMCs}$ for application in engines and nuclear reactors: an overview. Composites Science and Technology, 64(2), 155-170. doi: 10.1016/S0266-3538(03)00230-6

2. Krenkel W., Berndt F. (2005): C/C-SiC composites for space applications and advanced friction systems. Materials Science and Engineering: A, 412(1-2), 177-181. doi: 10.1016/j.msea.2005.08.204

3. Morscher G. N. (2004): Stress-dependent matrix cracking in $2 \mathrm{D}$ woven $\mathrm{SiC}$-fiber reinforced melt-infiltrated $\mathrm{SiC}$ matrix composites. Composites Science and Technology, 64(9), 1311-1319. doi: 10.1016/j.compscitech.2003.10.022

4. Yang C. P., Zhang L., Wang B., Huang T., Jiao G. Q. (2017): Tensile behavior of $2 \mathrm{D}-\mathrm{C} / \mathrm{SiC}$ composites at elevated temperatures: Experiment and modeling. Journal of the European Ceramic Society, 37(4), 1281-1290. doi: 10.1016/j.jeurceramsoc.2016.11.011

5. Camus G. (2000): Modelling of the mechanical behavior and damage processes of fibrous ceramic matrix composites: application to a 2-D $\mathrm{SiC} / \mathrm{SiC}$. International Journal of solids and Structures, 37(6), 919-942. doi: 10.1016/S00207683(99)00065-7

6. Yang Y., Xu F., Gao X., Liu G. (2016): Impact resistance of $2 \mathrm{D}$ plain-woven $\mathrm{C} / \mathrm{SiC}$ composites at high temperature. Materials \& Design, 90, 635-641. doi: 10.1016/j.matdes. 2015.11.024

7. Wang Y., Zhang L., Cheng L. (2013): Comparison of tensile behaviors of carbon/ceramic composites with various fiber architectures. International Journal of Applied Ceramic Technology, 10(2), 266-275. doi: 10.1111/j.1744-7402. 2011.02727.x

8. Sun Z., Wang C., Niu X., Song Y. (2016): A response surface approach for reliability analysis of $2.5 \mathrm{DC} / \mathrm{SiC}$ composites turbine blade. Composites Part B: Engineering, 85, 277-285. doi: 10.1016/j.compositesb.2015.09.025

9. Chen L., Yao X., Cen S. (2015): Predictions of elastic property on $2.5 \mathrm{DC} / \mathrm{SiC}$ composites based on numerical modeling and semi-analytical method. Composites Part B: Engineering, 74, 53-65. doi: 10.1016/j.compositesb.2015. 01.009 
10. Hallal A., Younes R., Fardoun F., Nehme S. (2012): Improved analytical model to predict the effective elastic properties of $2.5 \mathrm{D}$ interlock woven fabrics composite. Composite Structures, 94(10), 3009-3028. doi: 10.1016/j. compstruct.2012.03.019

11. Zhang C., Wang X., Liu Y., Wang B., Han D., Qiao S., Guo Y. (2013): Tensile fatigue of a $2.5 \mathrm{DC} / \mathrm{SiC}$ composite at room temperature and 900 C. Materials \& Design, 49, 814-819. doi: 10.1016/j.matdes.2013.01.076

12. Li Y., Xiao P., Luo H., Almeida R. S., Li Z., Zhou W., et al. (2016): Fatigue behavior and residual strength evolution of 2.5 DC/C-SiC composites. Journal of the European Ceramic Society, 36(16), 3977-3985. doi: 10.1016/j. jeurceramsoc.2016.07.009

13. Song J., Wen W., Cui H., Zhang H., Xu Y. (2016): Finite element analysis of $2.5 \mathrm{D}$ woven composites, part I: microstructure and 3D finite element model. Applied Composite Materials, 23(1), 29-44. doi: 10.1007/s10443-0159447-2
14. Lu Z., Zhou Y., Yang Z., Liu Q. (2013): Multi-scale finite element analysis of 2.5 D woven fabric composites under on-axis and off-axis tension. Computational Materials Science, 79,485-494.doi: 10.1016/j.commatsci.2013.07.003

15. Liu S., Tao L., Xie N.(2016): On the new model system and framework of grey system theory. Journal of Grey System, $28(1), 1-15$.

16. Rathnayaka R. K. T., Seneviratna D. M. K. N., Jianguo W. (2015): Grey system based novel approach for stock market forecasting. Grey Systems: Theory and Application, 5(2), 178-193. doi: 10.1108/GS-04-2015-0014

17. Evans M. (2014): An alternative approach to estimating the parameters of a generalised Grey Verhulst model: An application to steel intensity of use in the UK. Expert Systems with Applications, 41(4), 1236-1244. doi: 10.1016/ j.eswa.2013.08.006 